\title{
SONOGRAPHIC EXAMINATION OF THE EYEBALL USING WHOLE BODY USG EQUIPMENT IN CHILDREN: POTENTIAL AND CLINICAL SIGNIFICANCE
}

\author{
Jana Chmelová
}

\begin{abstract}
Radiodiagnostic Institute, Faculty of Health and Social Sciences, University of Ostrava, 70386 Ostrava - Zábřeh, Czech Republic, e-mail: jana.chmelova@fnspo.cz
\end{abstract}

Received: December 18, 2001

Key words: Sonography / Pediatric / Eyeball / Pathology of posterior pole

The aim of this study was to evaluate the potential of eyeball examination using whole body USG equipment for patients up to 18 years of age. The sonographic findings were compared with ophthalmoscopic results and clinical progress; follow-up sonographic examinations were carried out in many cases. Serious clinical states were operated and it was then possible to compare sonographic findings with surgical reports. Results obtained by other imaging methods, i. e. CT and/or MR examination, if these were carried out, were also used for comparison and for determination of the diagnostic yield of the sonographic examination of the eyeball. Using whole body USG equipment the most frequent pathological lesions of the posterior segment of the eyeball were unambiguously visualised. The results were statistically analysed. The results justify the thesis that the sonographic examination of the eyeball is the most useful, and most frequently, also the definitive imaging method for infant patients and its contribution, for example to differential-diagnostic judgments regarding vague findings on papilla, has a farreaching consequence. Last but not least, it is necessary to mention the cost-effectiveness of USG examination in comparison with both CT and MR examination.

\section{INTRODUCTION}

Ultrasound in ophthalmology was first used in the year 1956 by two American ophthalmologists, Mundt and Hughes ${ }^{1}$. In the sixties, imaging of the eyeball and orbit using ultrasound was popularised by the Australian ophthalmologist Ossoining ${ }^{2,3}$. In ordinary ophthalmological practice however use of ultrasound is still not widespread. This may be due to the high cost of specialized ophthalmologic USG equipment and their limited availability. Only little was written and mentioned about possibility to use whole body USG equipment which is designed for the examination of various organs. Results presented in this report indicate the potential of sonography in pediatric ophthalmology practice according to own experiences.

\section{MATERIALS AND METHODS}

\section{Technical details of investigation}

The investigated set includes sonographic examination of the eyeball in pediatric patients over the years 1993 through to 1999. Up to 1999 we used sonograpic equipment SONOLINE 2 (Siemens), with 7,5 MHz sector transducer. In 1999, patients were examined with SONOLINE VERSA PLUS (Siemens) with linear transducer at a frequency 7,5 MHz. To explore the anterior segment we used a stand - off for the elimination of the near field of the B image. We used the gel SONOPAD (CO ORD-MED CS) which is employed for sonographic examination of superficial layers. During examination the child is laying on his back with the head lightly inclined backwards. After applying a minimum quantity of ophthalmic ointment (Ophthalmo-Azulen) to the closed eyelid the transducer was applied. Then followed an indirect, transpalpebral examination. In the majority of cases, both eyes were examined without consulting the referring physician. If some control examination was needed, then we examined only the single eye. The findings were documented on multiformatted camera (Uni Quattro) or termoprinter (Mitsubishi). Using the Sonoline Versa equipment it was possible to save the data in digital format on diskette.

\section{Patient groups}

From 1. 5. 1993 to 31. 12. 1999 a total of 1468 USG eyeball examinations was made in 1101 patients. One examination implies a single patient not single eyeball. The investigated set includes 247 examinations in pediatric patients up to 18 years of age or $16,8 \%$ of the total. Indications for USG examination of the eyeball were determined by the ophthalmologists. Most frequently the patients were referred through the Eye Clinic and and the Department for squint and amblyopic children of the Faculty Hospital in Ostrava. 
Characteristics of the group:

Basic diseases (frequently not included in clinical reports) for which the children were followed by ophthalmologist

The most frequent basic disease for which children were followed and sent for was perinatal risk (52 investigations or 21\%) and eyeball trauma (38 investigations or $15,4 \%)$.

Ophtalmologic indications for USG examination (Table 1)

Table 1. Ophthalmologic indications

\begin{tabular}{|c|c|c|c|c|c|c|}
\hline \multicolumn{7}{|c|}{ Ophthalmologic indications } \\
\hline & $0-1$ & $1-5$ & $5-15$ & $15-18$ & Total & \\
\hline $\begin{array}{l}\text { pathology on the } \\
\text { papilla }\end{array}$ & -- & 12 & 47 & 12 & 71 & $28.7 \%$ \\
\hline $\begin{array}{l}\text { opaque ocular } \\
\text { media in } \\
\text { anterior segment }\end{array}$ & 7 & 9 & 23 & 6 & 45 & $18.2 \%$ \\
\hline $\begin{array}{l}\text { opaque ocular media } \\
\text { in posterior segment }\end{array}$ & --- & 7 & 19 & 8 & 34 & $13.8 \%$ \\
\hline $\begin{array}{l}\text { retinal changes - } \\
\text { other }\end{array}$ & 1 & 6 & 11 & 6 & 24 & $9.7 \%$ \\
\hline $\begin{array}{l}\text { retinal changes - } \\
\text { detachment }\end{array}$ & --- & 1 & 3 & 12 & 16 & $6.5 \%$ \\
\hline orbital patology & 4 & 5 & 6 & -- & 15 & $6.1 \%$ \\
\hline $\begin{array}{l}\text { retinal changes - } \\
\text { naevus }\end{array}$ & -- & -- & 1 & 5 & 6 & $2.4 \%$ \\
\hline not included & 11 & 10 & 13 & 2 & 36 & $14.6 \%$ \\
\hline Sum & 23 & 50 & 123 & 51 & 247 & \\
\hline
\end{tabular}

The most frequent indication for sonography was first vague finding on papilla of the optic nerve (71 examinations or $28 \%$ ), second opaque ocular media in the anterior segment (45 examinations or 18,2\%). In lower age groups it was caused mainly by congenital cataract, in older age groups by trauma.

\section{Ocular findings in individual groups of basic diseases}

In patients with perinatal risk the most frequent indication for sonographic examination was congenital cataract and suspicion of retinal detachment (13 examinations, $25 \%$ ). In patients with trauma history the most frequent indication was opaque ocular media in the anterior segment (blood in anterior chamber and corneal oedema), (14 examinations $36,7 \%$ ) and posterior segment (blood in the vitreous humour), (12 examinations or $31,6 \%$ ).

\section{Data analysis}

The sonographic findings were compared with ophthalmoscopic findings and clinical progress and with follow-up sonographic examinations. Serious clinical states required surgery and then it was possible to compare sonographic examination with the operation report. Results obtained by other imaging methods, i. e. CT and/or MR examination, if these were carried out, were also used for comparison and for determination of the diagnostic yield of the sonographic examination of the eyeball. The data were statistically analysed.

\section{RESULTS}

The results of sonographic examination of the eyeball were divided into the basic categories shown in Table 2.

Table 2. Results of sonographic examination of the eyeball

\begin{tabular}{|l|c|c|c|c|c|c|}
\hline \multicolumn{7}{|c|}{ Results of sonographic examination of the eyeball } \\
\hline & $0-1$ & $1-5$ & $5-15$ & $15-18$ & Total & \\
\hline normal finding & 19 & 27 & 51 & 14 & 111 & $44.9 \%$ \\
\hline $\begin{array}{l}\text { papillar } \\
\text { pathology }\end{array}$ & --- & 6 & 37 & 13 & 56 & $22.7 \%$ \\
\hline $\begin{array}{l}\text { changes in the } \\
\text { vitreous body }\end{array}$ & --- & 11 & 28 & 16 & 55 & $22.3 \%$ \\
\hline $\begin{array}{l}\text { retinal } \\
\text { detachment }\end{array}$ & --- & --- & 3 & 7 & 10 & $4.1 \%$ \\
\hline microphthalmus & 4 & 1 & --- & 1 & 6 & $2.4 \%$ \\
\hline other & --- & 5 & 4 & --- & 9 & $3.6 \%$ \\
\hline Sum & $\mathbf{2 3}$ & $\mathbf{5 0}$ & $\mathbf{1 2 3}$ & $\mathbf{5 1}$ & $\mathbf{2 4 7}$ & \\
\hline
\end{tabular}

In 111 examinations, sonographic findings of the posterior pole were normal, the area of the vitreous humour was completely anechogenic, it was surrounded by the hyperechogenic eyeball wall (Fig. 1). Pathological changes in vitreous humour were found in 55 cases. Most frequently this was bleeding and postoperative changes $(23 \times)$, proliferative changes in the vitreous humour, connected with retinopathy of the preterm $14 \times$ (these always agreed with ophthalmoscopic findings), hemophthalmus $8 \times$ (Fig. 2), inflammatory changes in vitreous humour $7 \times$ (Fig. 3), manifestation of endophthalmiditis $3 \times$. Inflammation was in 5 cases confirmed laboratory. CT examination was performed three times, always with negative results (no changes in vitreous humour were detected on the image), that is, the CT scan gave $3 \times$ false negative results. MR investigation was used twice, and showed changes in vitreous humour, confirmed sonograhic findings but otherwise made no further diagnostic contribution.

Definite findings of drusen on the papilla of the optic nerve, displayed as hyperechogenicity in the region of the optic nerve exit with pronounced acoustic shadow, was determined $51 \times$ (Fig. 4). Eodema of the papilla (Fig. 5) which sonography showed as hyperechogenic prominence in the region of the optic nerve papilla without acoustic shadow, was diagnosed $5 \times$. This finding was verified by follow-up examinations, when after employing anti-eodema therapy the finding has normalised in confirmation with ophthalmoscopic findings. Retinal detachment was diagnosed $10 \times$ (Fig. 6). In five cases the small patients were operated and the sonography results were confirmed by the surgeon. Microphthalmus was found in six cases, other findings in nine examinations (Fig. 7).

The youngest of 23 patients up to one year of age was two days old. Seven children were investigated for opaque ocular media in the anterior segment (congenital cataract), the other in connection with retinopathy of the 
preterm. Through sonography we found 4 cases of microphthalmus, in the other cases the sonographic findings were normal. Children with cataract were operated and during the operation no other eyeball disorder was discovered.

50 investigations of preschool children aged $1-5$ years were made in total. Sonography was evaluated as normal in 27 examinations, changes in the vitreous humour were found in 11 cases, findings on the papilla of the optic nerve in total $6 \times$. Three times were evaluated as drusen on the papilla and three times as eodema of the papilla. These results were always confirmed by clinical progress and control USG examinations. Half the cases, $(25 \times)$ were originally preterm infants being followed for different expressed stages of retinopathy of the premature.

School children formed nearly half of the investigated set (123). In these patients basic diagnosis were frequently not included $(73 \times), 23 \times$ the children were referred with diagnose trauma, $16 \times$ they were examined for disorder of eyeball movement. Young people aged 16-18 formed a group of 51 investigations. Trauma of the eyeball was studied in 15 cases, nine patients needed sonography for perinatal risk and disorder of eyeball movement.

For trauma of the eyeball 38 investigations in total were completed in 27 children ( 24 boys, 3 girls). Perforation injury of the eyeball was the reason of the examination in 4 cases, contusion of the eyeball was diagnosed by the ophthalmologist $20 \times$. In three children the type of injury was not announced. Enquiries concerning the causes of the prevalence of trauma in boys were not unsurprisingly; fighting $(5 \times)$, blow from sports instrument $(5 \times)$, wide range of objects (bottle caps, aronia berries) insults $(5 \times)$, work injuries $(2 \times)$, firearm accidents $(2 \times)$. The reason for sonographic investigation of the eye was most commonly opaque ocular media $(26 \times)$ and suspicion of retinal detachment $(6 \times)$. The sonographic results were normal in 15 cases, a diagnosis of retinal detachment was made in 6 cases, of which $3 \times$ it was a new finding in the eye with hemophthlamus. Changes in the vitreous humour were diagnosed $17 \times$, of which in one case it was established as subluxed lens (Fig. 8). The CT examination of the eye and orbit in connection with brain scan was followed in 5 patients, without producing new information about eyeball defects. Seven children were operated on and the operation protocols showed results confirmed sonographic findings.

\section{Correlation of acquired results with other findings}

Sonographic examination was always the first method of choice. CT examination of the eyeball was realized in 8 patients. In five small patients the results corresponed to sonographic findings, in 3 cases the CT results failed to display findings noted by sonography, that is, the CT scan gave three false negatives. MR examination was performed two-times, both cases confirmed sono- graphic findings. Following the sonography in total 18 children were operated. Surgical reports confirmed sonographic findings.

\section{DISCUSSION}

Sonographic examination unambiguously deserves the foremost position in the algorithm for imaging pathological states of the eyeball because of the absence of radiation risk, simplicity, rapid result, a good availability and low cost. In the examination of infants and newborn children an important role plays the absence of anesthesia which must be applied during CT and MR examinations. A further advantage of sonography compared with CT and MR examination is the fact that in sonography of the eye it is possible to image the movement of different pathological structures, what is a very important phenomenon for the differential diagnostic considerations. It deals above all with imaging of pathological states of the vitreous humour and retinal detachment or ablation of chorioid. In the algorithmus for imaging ocular structures in pediatric patients the whole body USG equipment holds principle position because of its possibility to create high quality images of the posterior pole of the eyeball and to spare the children of further examinations. The basic contribution of sonography is diferential-diagnostic judgments regarding vague findings on papilla of the optic nerve ${ }^{6,7,8}$. Determining a diagnosis of optic nerve drusen excludes oedema of the brain and, of course, all diagnostic procedures which are connected with this patological state ${ }^{9,10}$.

MR examination is the second in algorithm for imaging pathological states of the eyeball. It is irreplaceable in imaging patological states of the eyeball, for detecting and diagnostics changes in the eyeball wall and in the imaging of tumour spreading ${ }^{11,12,13,14}$. MR examination can easy and distinctly image vitreous opacities although, as in the case of sonography, it does not contribute to the cause of this state. The value of the verdict from the MR examination for the assessment of pathological conditions of the eyeball, is higher than CT examination. Disadvantages of MR examination include length of the investigation (20 mins), its poor availability and also the cost of the examination. MR examination is expensive and its cost is raising using the special induction coil for eyeball investigation, and finally using the contrast medium ${ }^{15}$. Another disadvantage is also influence of spontaneous movements of the eye which lead to annoying movement artefacts. Suspected presence of metal foreign bodies in the eyeball or in the orbit are one of the contraindications against MR imaging not only eyball and orbit but also CNS. During MR examination the intraocular metal foreign body may shift by 7-10 $\mathrm{mm}$, and this can lead to serious injury to ocular structures ${ }^{16,17,18}$.

In the case of CT examination of the eyeball it is necessary to bear in mind the radiation risk of the eye lens which is a highly radiosensitive organ. On top of 
that is the fact that it is necessary to repeate the examination with application of contrast medium i. v. (using of helical method scanning provides the possibility for lowering the risk). The basic principle of radiation protection in examination of the eyeball and orbit, is the justification for its use and this must be born constantly in mind. More often than in other indications, it is necessary to consult the referring physicians about the expected diagnosic contributions and if there is ambiguity, to propose another imaging method which can give the required information without radiation burden for the patient. A further disadvantage of CT exmination is the proportionally small difference in the density of vitreous body and the eyeball wall, making it impossible to image fine lesions. Intravenous application of the contrast medium does not contribute to result, because the exact measurement density of small lesions is not possible. Further negative effects (allergy, kidney failure) are connected with the application of contrast medium i. v. During the examination of pediatric patients, the side effects of anesthesia are also necessary to be taken in account.

\section{CONCLUSIONS}

Sonographic examination unambiguously deserves the foremost position in the algorithm for imaging pathological states of the eyeball because of its effective contribution to the diagnose, and as a result, subsequent therapy of eye diseases without increasing the exposure of sick people to radiation. The results justify the thesis that the sonographic examination of the eyeball is the most useful, and most frequently, also the definitive imaging method for infant patients, and its contribution, for example to differential-diagnostic judgments regarding vague findings on papilla, has a far-reaching consequence. Last but not least, it is necessary to mention the cost-effectiveness of USG examination in comparison with both $\mathrm{CT}$ and MR examinations.

\section{REFERENCES}

1. Mundt, G. H. jr., Hughes, W. F. jr. (1956) Ultrasonics in ocular diagnosis. Amer. J. Ophthalmol. 41, 488.

2. Ossoinig, K. C. (1979) Standardized echography: Basic principles, clinical applications and results. Int. Ophthalmol. Clin. 19, 127.

3. Ossoinig, K. C. (1985) Standardized ophthalmic echography of the eye, orbit and periorbital region. A comprehensive slide set and study guide. Iowa City, Goodfellow.

4. Chmelová, J. (1996) Diagnostický přínos sonografického vyšetření očního bulbu. Čes. Radiol. 4, 261-265.

5. Chudáček, Z. jr., Hradecká, V. (1992) Ultrasonografický obraz luxace jádra oční čočky. Čes. Radiol. 46, 370-372.

6. Ballantyne, J., Hollman, A. S., Hamilton, R., Bradnam, M. S., Carachi, R., Young, D. G., Dutton, G. N. (1999) Transorbital optic nerve sheath ultrasonography in normal children. Clin. Radiology 54, 740-742.

7. Balmer, A., Munier, F., Uffer, S. (1998) Diagnostic imaging of intraocular lesions in the child. Klin. Monatsbl. Augenheilk. 212, 252-256.

8. Balmer, A., Munier, F. (1999) Leukokoria in a child: emergency and challenge. Monatsbl. Augenheilk. 214, 332-335.

9. Long, G., Stringer, D. A., Nadel, H. R., Fink, A. M., Lewis, P., Carruthers, J. D., Lyons, C. (1998) B mode ultrasonography spectrum of paediatric ocular diseases. Eur. J. Radiol. 26, 132-147.

10. Panarello, S. M., Priolo, E., Vittone, P. (1998) Pediatric ultrasound a personal experience during the period 1991-1994. Ophthalmologica 212, 115-117.

11. DePotter, P., Flanders, A. E. P., Shields, J. A., Shields, C. L. (1993) Magnetic resonance imaging of intraocular tumors. Int. Ophthalmol. Clin. 33, 37-45.

12. DePotter, P., Shields, J. A., Shields, C. L. (1995) Tumors of the uvea in: MRI of eye and orbit (DePotter, P., Shields, J. A., Shields, C. L), Philadelphia, Lippincott, pp. 55-92.

13. Keizer, R. J. W., Vielvoye, G. J., Wolff-Rouendaal, D. (1986) Nuclear magnetic resonance imaging of intraocular tumors. Amer. J. Ophthalmol. 102, 438-441.

14. Raymond, W. R., Char, D. H., Norman, D., Protzko, E. E. (1991) Magnetic resonance imaging evaluation of uveal tumors. Amer. J. Ophthalmol. 111, 633-641.

15. Mihara, F., Gupta, K. L., Murayma, S. (1991) MR imaging uveal melanoma. Role of pulse sequence and contrast agent. Amer. J. Neuroradiol. 12, 991-96.

16. Kelsey, C. A., King, J. N., Keck, G. M., Chiu, M. T., Wolfe, D. M., Orrison, W. W. (1991) Ocular hazard of metallic fragments during MR imaging at 0,06 Tesla. Radiology 180, 282-283.

17. Kremmer, S., Schiefer, U., Wilhelm, H., Zrenner, E. (1996) Mobilisation intraokularer Fremdkörper durch Magnet-Rezonanz-Tomographie. Klin. Monatsbl. Augenheilk. 208, 201-202.

18. Ta, Ch. N., Bowman, R. W. (2000) Hyphema caused by a metallic intraocular foreign body during magnetic resonance imaging. Amer. J. Ophthalmol. 129, 533-534. 
Sonographic examination of the eyeball using whole body USG equipment in children: potential and clinical significance

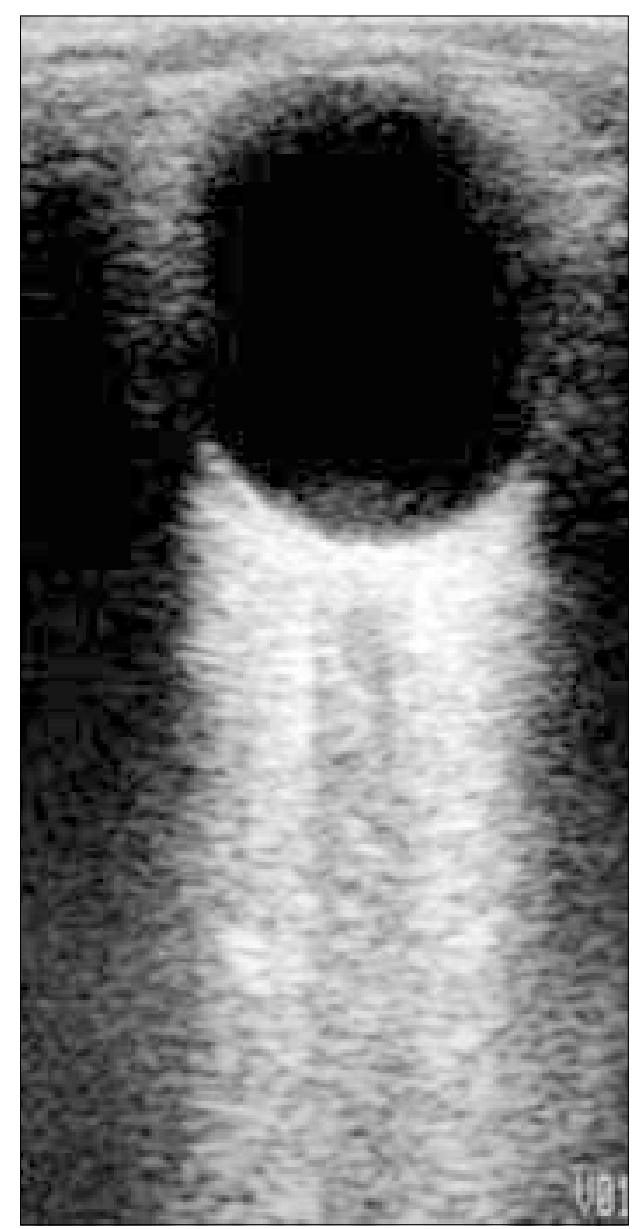

Fig. 1 Normal sonographic finding in vitreous body

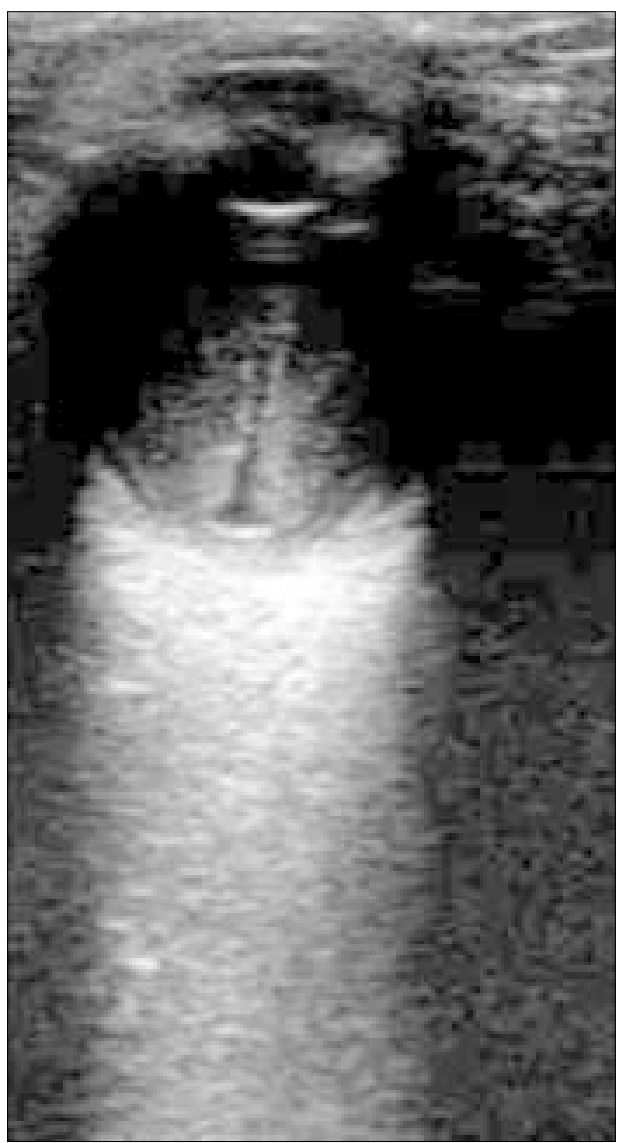

Fig. 2 Vitreous hemorrhage secondary to Terson's syndrom - the region of vitreous body is filled by small dense opacities

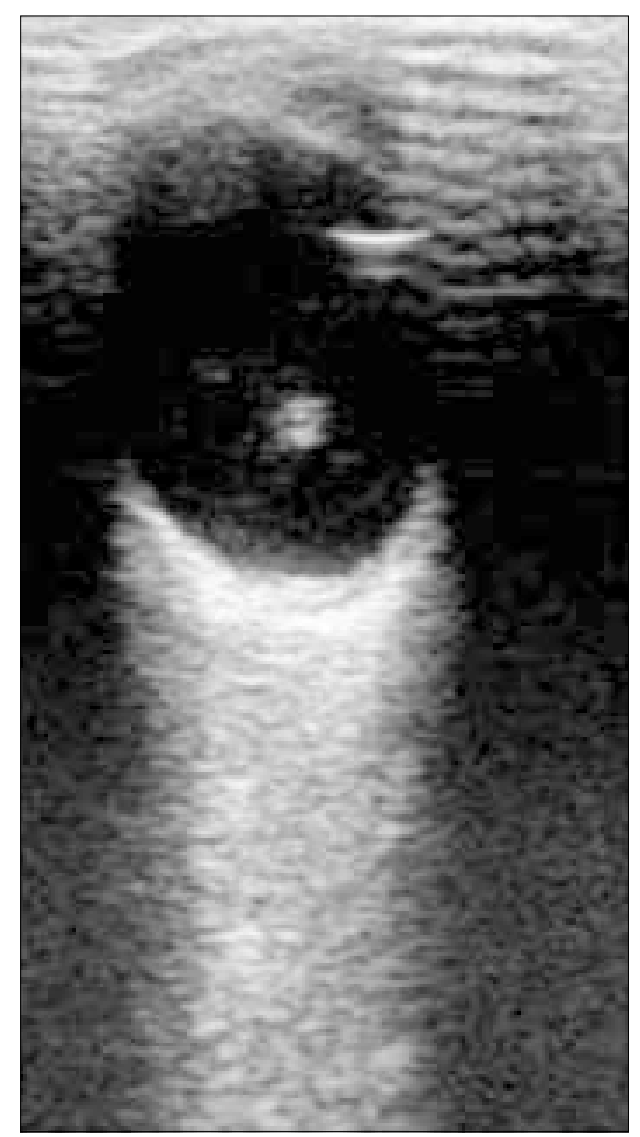

Fig. 3 Opacities in vitreous body by toxoplasmosis

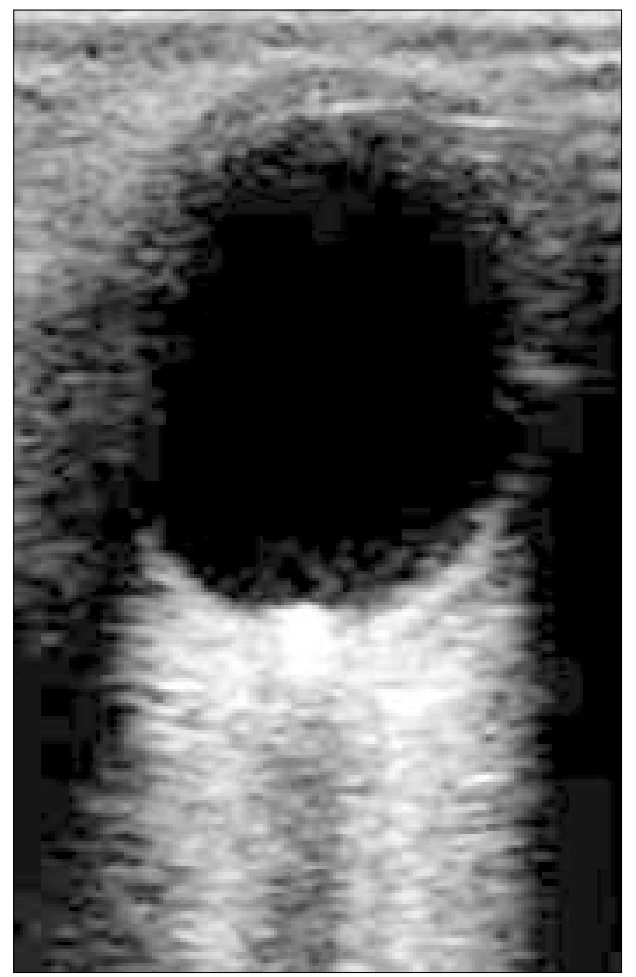

Fig. 4 Drusen on the papilla of the optic nerve 


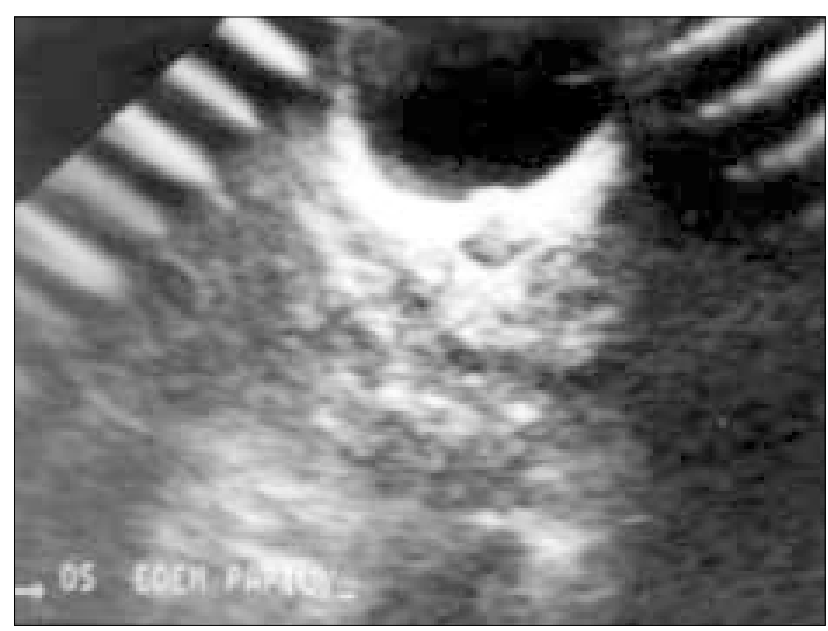

Fig. 5 Oedema of the papilla of the optic nerve

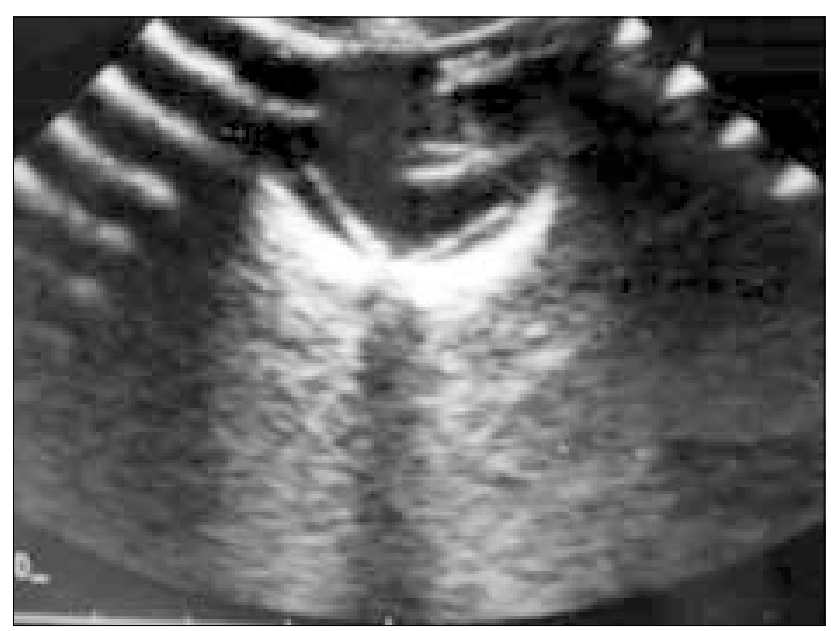

Fig. 6 Retinal detachment at the posterior pole

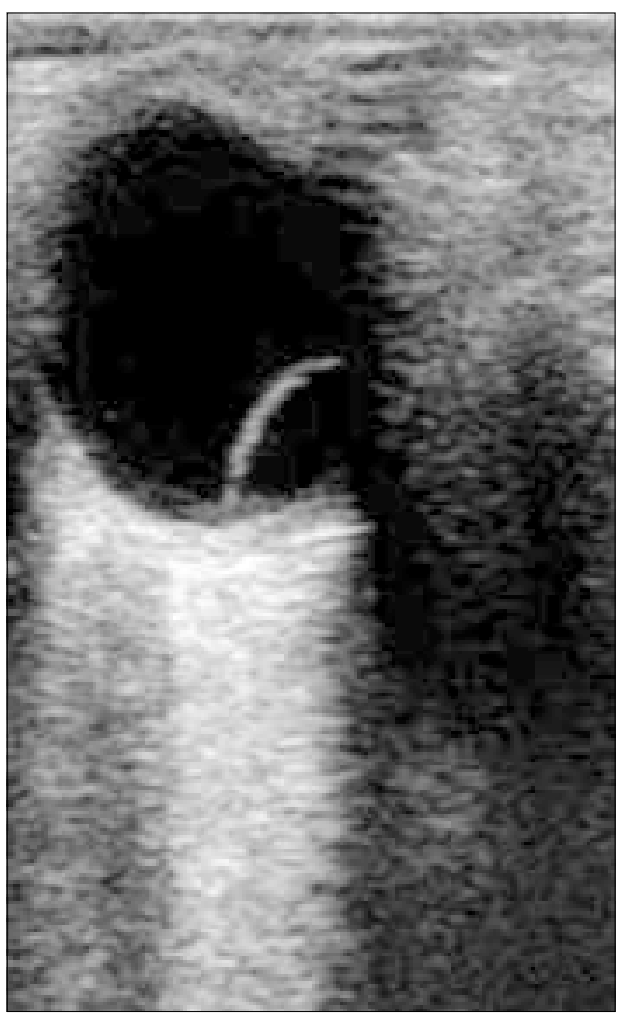

Fig. 7 Retinoschisis - moderately elevated, thin membrane in the periphery

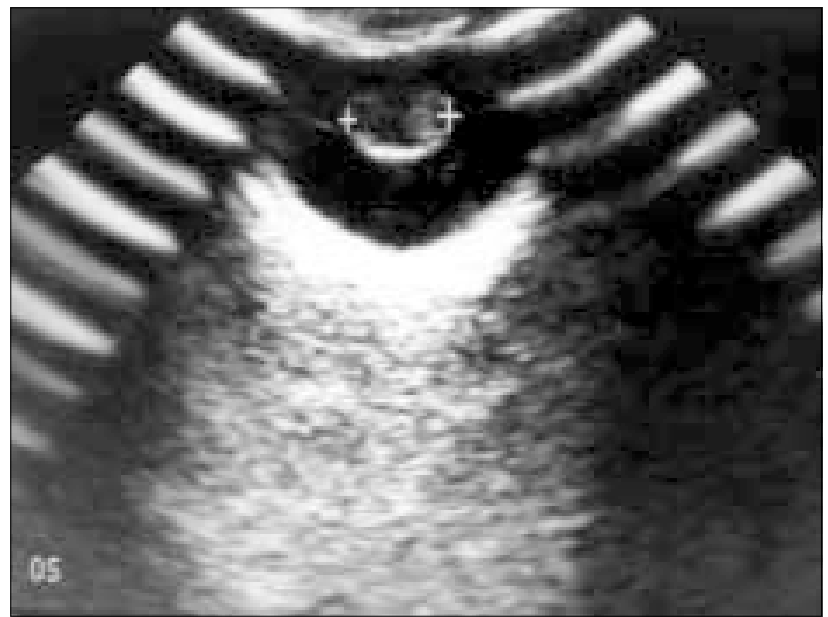

Fig. 8 Subluxated lens 